\title{
Comparison of Different Surface Modification Techniques for Electrodes by Means of Electrochemistry and Micro Synchrotron Radiation X-ray Fluorescence. Dimerization of Cobalt(II) Tetrasulfonated Phthalocyanine and Its Influence on the Electrodeposition on Gold Surfaces
}

\author{
Karl Peeters, Karolien De Wael,* Laszlo Vincze, and Annemie Adriaens \\ Department of Analytical Chemistry, Ghent University, Krijgslaan 281 S12, B-9000 Ghent, Belgium
}

This paper compares different electrochemical surface modification techniques with special attention to the immobilization of cobalt(II) tetrasulfonated phthalocyanine tetrasodium salt (Co(II)TSPc) on gold electrodes. Electrochemical and synchrotron radiation X-ray fluorescence (SR-XRF) microbeam analysis were performed in order to compare the amount of adsorbed CoTSPc onto the gold electrode and to determine the level of uniformity of the deposited layer. The nondestructive, quantitative characterization of CoTSPc deposition on gold electrodes by means of scanning SR-XRF on the microscopic scale has never been described before. The described methodology can be in general used for thin-film characterization. Depending on the degree of dimerization of the CoTSPc molecules, different electrochemical behavior is observed.

Since their discovery in 1934 by Linstead, ${ }^{1}$ phthalocyanines and their derivatives have been widely used as dyestuffs and colors. Nowadays they are also commonly used in high-tech applications, such as photovoltaic, electronic, and sensing devices. ${ }^{2-6}$ The major characteristics of phthalocyanines, frequently used in electrocatalysis, ${ }^{7-8}$ are their thermal and chemical stability, coupled with their extensive redox chemistry.

More than 70 different metal ions can form a complex by coordinating with the phthalocyanine ring. ${ }^{1}$ The aromatic ring structure can be oxidized, reduced, or both. If the central metal ion is a transition element, it can undergo oxidation or reduction at potentials comparable to those of the ring processes. ${ }^{1}$

* Corresponding author. Tel: +32 926448 20. Fax: +32 92644960 . E-mail: Karolien.DeWael@UGent.be.

(1) Linstead, R. P. J. Chem. Soc. 1934, 1016

(2) Leznoff, C. C.; Lever, A. B. P. Phthalocyanines Properties and Applications; VCH Publishers: Weinheim, 1993; Vol. 3.

(3) Lever, A. B. P. Chemtech 1987, 17, 506-510.

(4) Kato, M.; Nishioka, Y.; Kaifu, K.; Kawamura, K.; Ohno, S. Appl. Chem. Lett. 1985, 86, 196-197.

(5) Kuder, J. E. J. Imaging Sci. 1988, 32, 51-56.

(6) Temofonte, T. A.; Schoch, K. F. J. Appl. Phys. 1989, 65, 1350-1355.

(7) Balkus, K. J.; Eissa, M.; Levado, R. J. Am. Chem. Soc. 1995, 117, 1075310754.

(8) Andreev, A.; Ivanova, V.; Prahov L.; Schopov, I. D. J. Mol. Catal. 1995, 95, 197-201.
The aromatic ring structure is completely flat, which enables the phthalocyanines in the deposited layer to form columnar aggregates, in which $\pi$-stacking interactions between the molecules dominate. It is desirable to have ordered, well-packed, and well-oriented molecular layers of phthalocyanines on the electrodes. The aggregation of phthalocyanines has been the subject of intensive study over many decades. ${ }^{9-11}$ The degree of aggregation can have significant effects on, for example, the light fastness and color quality of dyestuffs. Another example is the observation that aggregates are much less active than monomers concerning the use of phthalocyanines as photosensitizers in photodynamic cancer therapy.

Soluble sulfonated phthalocyanines have been shown to be able to form layers on different types of supporting material, including electrodes. The immobilization of these compounds on an electrode can lead to modified electrodes with important electrocatalytic properties. $^{12}$

This article focuses on the immobilization characteristics of cobalt(II) tetrasulfonated phthalocyanine tetrasodium salt (Co(II)TSPc) on gold electrodes. Different electrochemical techniques are described to produce CoTSPc-modified gold electrodes, such as the immersion of the electrode in a Co(II)TSPc solution, the drop drying, and the potential cycling methods. In the first part of the paper, a detailed comparison is made between the abovementioned techniques with respect to the resulting Co thin-film properties on the electrode surface, based on electrochemical and scanning micro synchrotron radiation X-ray fluorescence (SR-XRF) measurements.

In the second part of the paper, the degree of aggregation is discussed, which was studied by performing UV-visible absorption spectroscopy analyses. A remarkable observation was the difference in dimer-monomer equilibrium for the $\mathrm{Co}$ (II)TSPc samples purchased from different companies. The influence of

(9) Yang, Y.-C.; Ward, J. R.; Seiders, R. P. Inorg. Chem. 1985, 24, 1765-1769. (10) Thamae, M.; Nyokong, T. Polyhedron 2002, 21, 133-140.

(11) Camp, P. J.; Jones, A. C.; Neely, R. K., Speirs, N. M.J. Phys. Chem. A 2002, 106, 10725-10732.

(12) De Wael,K.; Westbroek, P.; Temmerman, E. Electroanalysis 2005, 17 (3), 263-268.

10.1021/ac0508245 CCC: $\$ 30.25$ @ 2005 American Chemical Society Published on Web 07/14/2005 
the dimerization of $\mathrm{Co}$ (II)TSPc on its electrochemical behavior at gold electrodes is described in the last section of this article.

\section{EXPERIMENTAL SECTION}

A saturated calomel reference electrode (SCE) with two compartments from Radiometer (Analis, Belgium) and a carbon counter electrode were used. The working electrodes were gold electrodes from BAS, having a diameter of $1.6 \mathrm{~mm}$ (Bioanalytical Systems Inc.), which were pretreated by mechanical and electrochemical polishing. Before its first use, the electrode surface was scoured briefly on SiC-emery paper, 1200 grit, to obtain a fresh surface. To reduce the relatively high surface roughness, the electrode was further subjected to sequential polishing using a polishing cloth covered with alumina (Buehler) powder of 1-, 0.3-, and $0.05-\mu \mathrm{m}$ particle size for, respectively, 5,10 , and $20 \mathrm{~min}$. To remove any adherent $\mathrm{Al}_{2} \mathrm{O}_{3}$ particles, the electrode surface was rinsed thoroughly with doubly deionized water and cleaned in an ultrasonic bath (Branson 3210) for 2 min. Finally, the electrode was pretreated electrochemically by scanning it in a $\mathrm{Na}_{2} \mathrm{HPO}_{4}$ / $\mathrm{NaOH}$ buffer solution ( $\mathrm{pH} 12$, purchased from Riedel-de Haën) between -1.2 and $0.6 \mathrm{~V}$ versus SCE until five subsequent scans were identical. This pretreatment was done to smooth the gold surface and the remove any oxides.

A PGSTAT20 potentiostat (ECO Chemie) controlled by GPES 4.9 software package running on a Pentium II computer (Eknadata) was used to record the voltammetric curves. Measurement of the solution $\mathrm{pH}$ was done using an Orion Benchtop pH-meter model 420A. Before each experiment, pure nitrogen was bubbled through the cell solution for $20 \mathrm{~min}$.

The Co(II) tetrasulfonated phthalocyanine sodium salts were purchased from Midcentury (Posen), ${ }^{13}$ Porphyrin Systems (Luibeck), and Rhodes University of Grahamstown (Eastern Cape, South Africa). ${ }^{13}$

UV-visible measurements were performed with a Varian Cary $3 \mathrm{E}$ spectrophotometer (Instrument version 8.01) (Palo Alto, CA).

Electrochemical Modification Techniques. The first modification technique ("immersion method") involved the immersion of a gold electrode in a $6 \times 10^{-3} \mathrm{~mol} \mathrm{~L}^{-1} \mathrm{Co}$ (II)TSPc $\mathrm{pH} 12$ buffer solution for $100 \mathrm{~min}$. After the modification, the electrode was scanned in a pH 12 buffer solution not containing Co(II)TSPc in order to study the electrochemical behavior of the modified electrode and to obtain information about the amount of CoTSPc deposited on the gold surface. Figure 1a shows a few of the 20 cyclic voltammetric scans measured in the blank pH 12 buffer solution.

In the second technique ("drop-drying method"), the gold electrode was modified by depositing a droplet of the $6 \times 10^{-3}$ mol L ${ }^{-1}$ Co(II)TSPc pH 12 buffer solution on its surface. When the solution was dried, the modified electrode was scanned in a pH 12 buffer solution containing no Co(II)TSPc. Figure 1b represents a few of the 20 cyclic voltammetric scans recorded in the buffer solution after the drop drying process.

During the third modification procedure ("potential cycling method"), a gold electrode was modified with CoTSPc by recording successive cyclic voltammetric scans (100 scans) in a potential window from -1.2 to $0.6 \mathrm{~V}$ versus SCE in a $\mathrm{pH} 12$ buffer solution containing $6 \times 10^{-3} \mathrm{~mol} \mathrm{~L}^{-1} \mathrm{Co}$ (II)TSPc. After completion of the

(13) Weber J. H.; Busch D. H. Inorg. Chem. 1965, 4, 469. electrochemical deposition experiment, the modified electrode was further scanned in the buffer solution in the absence of $\mathrm{Co}$ (II)TSPc. A few of the first 20 voltammetric scans are presented in Figure 1c. A detailed explanation of all electrochemical processes can be found in the literature. ${ }^{14-16}$

Scanning SR-XRF. Scanning micro SR-XRF experiments were performed at Beam Line L of the DORIS-III storage ring, HASYLAB (Hamburg, Germany). ${ }^{17}$ This beamline is dedicated to micro XRF experiments using either white or monochromatic bending magnet excitation and mono- or polycapillary focusing, with routinely available beam sizes of $10-30 \mu \mathrm{m}$. Figure 2 shows the experimental arrangement used during our scanning micro XRF experiments, which also allowed optional X-ray absorption near edge structure spectroscopic measurements in fluorescence or in transmission mode.

The primary X-ray beam is generated by a $1.2 \mathrm{~T}$ bending magnet source, which, given the machine energy of $4.465 \mathrm{GeV}$, provides a polychromatic spectral distribution with a critical energy of $16.6 \mathrm{keV}$. After initial low-energy filtering and collimation, the beam was monochromatized by a $\mathrm{Si}(111)$ fixed-exit monochromator $\left(\Delta E / E \approx 10^{-4}\right)$ and further collimated by a motorized cross-slit system. After monochromatization, the beam was focused by a monolithic polycapillary half-lens (X-ray Optical Systems Inc.), designed specifically for parallel primary beams and hence suitable for the focusing of low-divergence synchrotron beams. A microbeam size of $\sim 20 \mu \mathrm{m}$ (FWHM) was obtained at the excitation energy range of $7.7-8.0 \mathrm{keV}$, which was used to excite selectively the $\mathrm{Co} \mathrm{K} \alpha, \beta$ fluorescent lines without exciting $\mathrm{Au}$ L lines from the (spectroscopically infinitely thick) gold substrate. The nominal working distance of the polycapillary optic was $5 \mathrm{~mm}$.

Experimental difficulty of the CoTSPc thin-film uniformity studies included the determination of low amounts of Co $(\sim 0.1-$ $0.5 \mathrm{pg}$, assuming a $20 \mu \mathrm{m}$ incident beam) in the presence of partially overlapping Compton and Au X-ray resonant Raman peaks. During the CoTSPc uniformity studies, $21 \times 21$ or $41 \times$ 41 scanning XRF maps were recorded with typically $20-\mu \mathrm{m}$ step size, using a data collection time of $10-20 \mathrm{~s}$ per data point. Absolute detection limits for Co were estimated to be $7-8 \mathrm{ng} \mathrm{cm}^{-2}$ using a measuring time of $20 \mathrm{~s}$. To determine the level of uniformity of the deposited CoTSPs thin film, the scanning and subsequent data reduction strategy described by Kempenaers et al. has been applied. ${ }^{18}$

\section{RESULTS AND DISCUSSION}

Comparison of Different Electrochemical Modification Techniques. The data in Figure 1a, corresponding to the electrode immersion method, show the growth of the $\mathrm{Co}(\mathrm{III})_{\text {ads }} /$ $\mathrm{Co}(\mathrm{II})_{\text {ads }}$ redox couple during the first 20 voltammetric scans in the buffer solution after the modification, which implies that some

(14) De Wael, K.; Westbroek, P.; Temmerman, E. J. Electroanal. Chem. 2004, 567, 167-173

(15) De Wael,K.; Westbroek, P.; Bultinck, P.; Depla, D.; Vandenabeele, P.; Adriaens, A.; Temmerman, E. Electrochem. Commun. 2005, 7, 87-96.

(16) De Wael,K.; Westbroek, P.; Adriaens, A.; Temmerman, E. Electrochem. SolidState Lett. 2005, 8 (4), C65-C68.

(17) Falkenberg, G.; Clauss, O.; Swiderski, A.; Tschentscher T. X-Ray Spectrom. 2001, 30 (3), 170-173.

(18) Kempenaers, L.; Janssens, K.; Vincze, L.; Vekemans, B.; Somogyi, A.; Drakopoulos, M.; Simionovici, A.; Adams, F.; Anal. Chem. 2002, 74 (19), 5017-5026. 

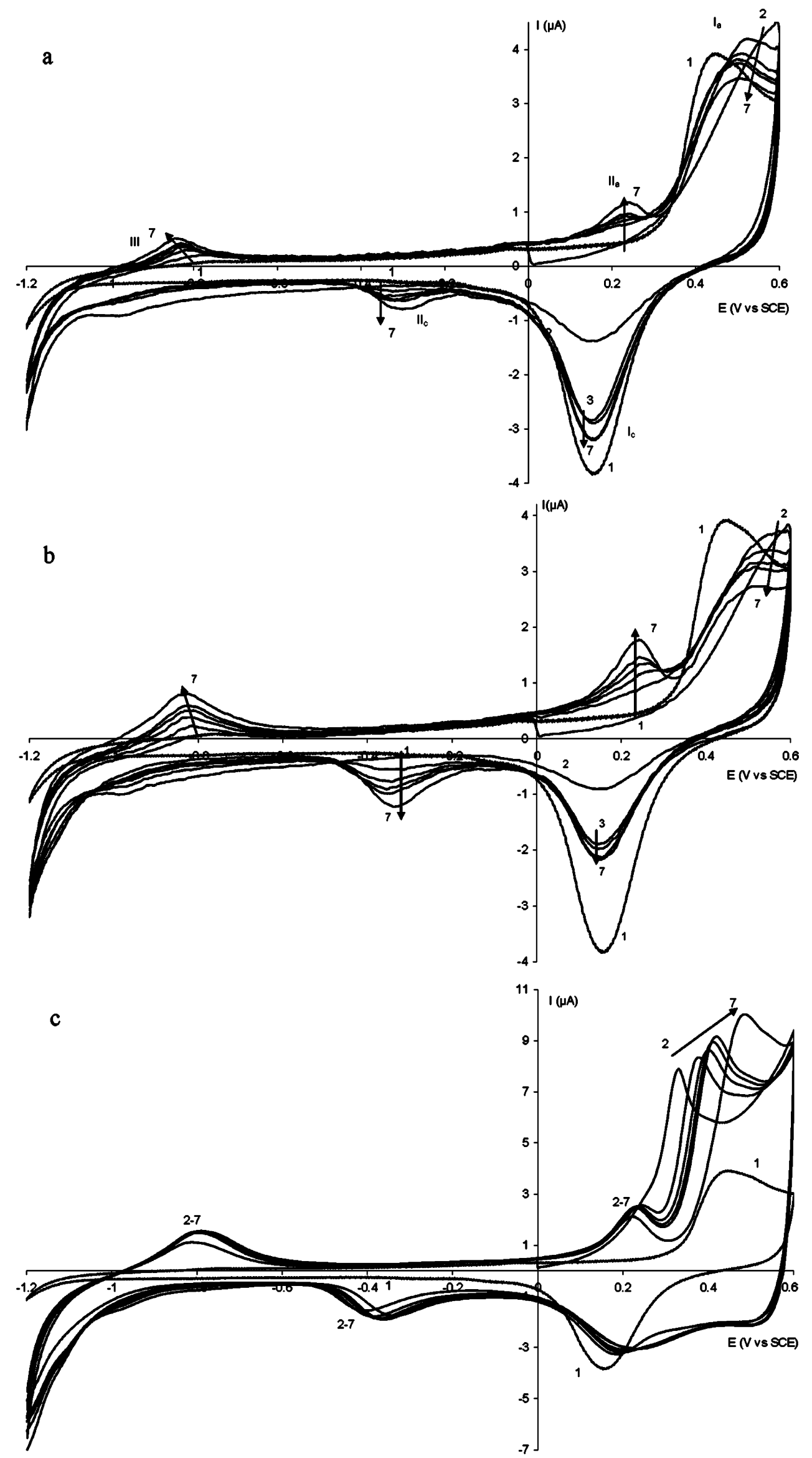

Figure 1. Current-potential curves recorded at a gold electrode in a pH 12 buffer solution (1) and scanning of the modified electrode in a pH 12 buffer solution (2-7). Scan numbers: (2) 1, (3) 3, (4) 5, (5) 10, and (6) 20. Different modification techniques: electrode immersion (a), drop drying (b), and potential cycling (c). 

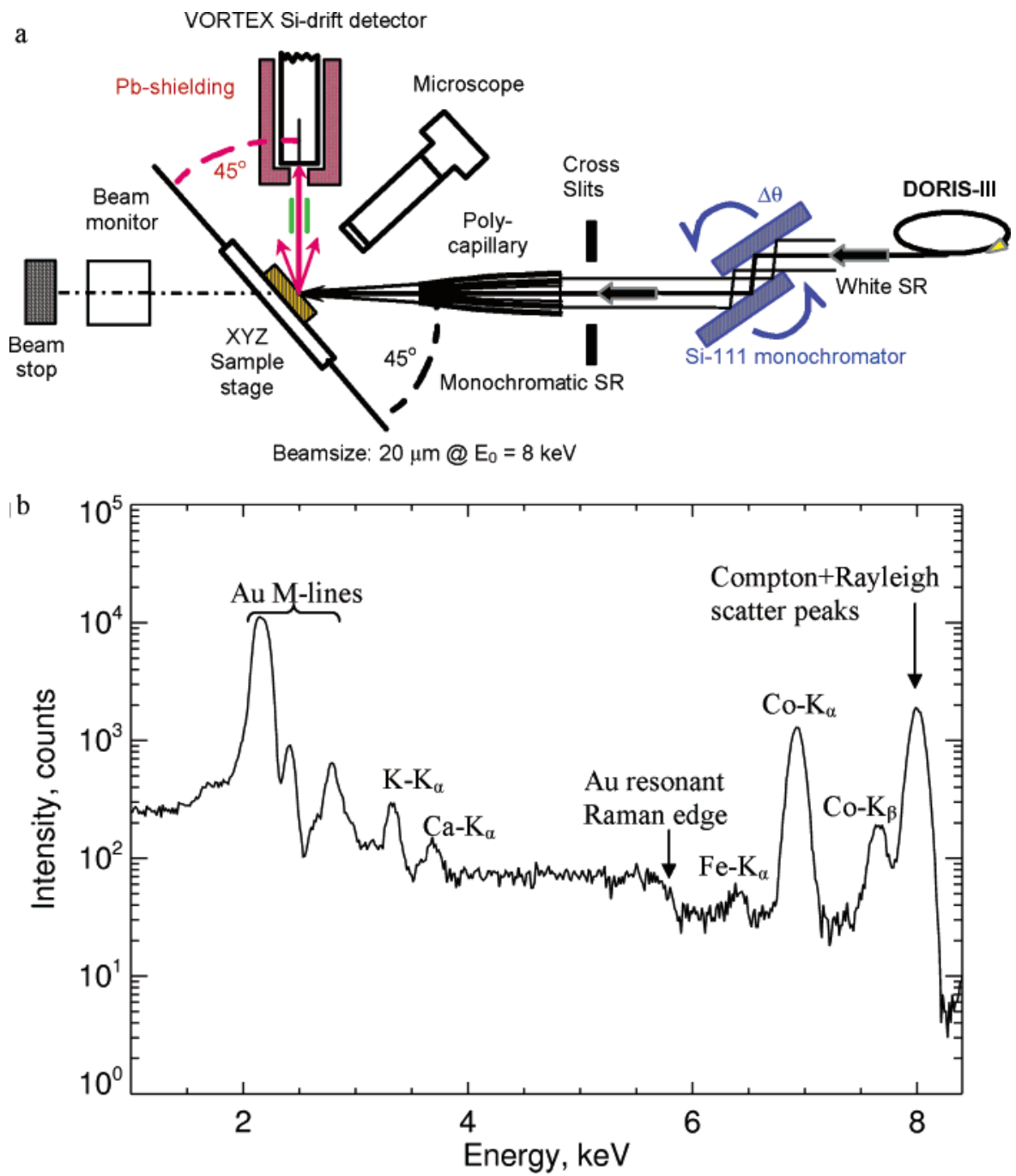

Figure 2. Schematic representation of the micro SR-XRF instrument installed at HASYLAB Beam Line L (Hamburg, Germany) (a) and a typical XRF spectrum measured at this instrument for a gold electrode corresponding to a measuring time of $500 \mathrm{~s}$ (b).

of the CoTSPc is adsorbed onto the gold surface. The peaks at $-0.335\left(\mathrm{II}_{\mathrm{c}}\right)$ and $0.236 \mathrm{~V}\left(\mathrm{II}_{\mathrm{a}}\right)$ versus SCE reflect the reduction/ oxidation behavior of adsorbed $\mathrm{Co}$ (III)TSPc and Co(II)TSPc. The peaks attributed to a ring reaction of adsorbed CoTSPc appear at $\sim-0.9 \mathrm{~V}$ versus SCE and are indicated by III in Figure 1a.

Another observation that provides evidence for adsorption is the decrease of the relatively broad $\mathrm{AuO}$ formation peak around $0.5 \mathrm{~V}$ versus $\mathrm{SCE}\left(\mathrm{I}_{\mathrm{a}}\right)$ and its related reduction signal at $0.15 \mathrm{~V}$ versus SCE $\left(\mathrm{I}_{\mathrm{C}}\right) .{ }^{19-22}$ As the gold surface is covered with CoTSPc, the oxidation and reduction of the gold(oxide) surface is prevented. A remarkable result is the growth of the CoTSPc-related adsorption peaks during the first 10 scans. After these 10 scans, a stable cyclic voltammogram is observed. This growth with increasing scan number is probably associated with the transformation of a heterogeneous surface layer into a homogeneous surface condition as indicated by the scanning SR-XRF uniformity studies discussed below. The charge under the $\mathrm{Co}$ (III)/Co(II) adsorption reduction peak, based on scan 20 , is equal to $2.4 \pm$

(19) Burke, L. D.; O’Leary, W. A. J. Appl. Electrochem. 1989, 19, 758-767.

(20) Burke, L. D.; O'Sullivan, J. F. Electrochim. Acta 1992, 37(4), 585-594.

(21) Woods, R. In Electroanalytical Chemistry; Bard, A. J., ed.; Marcel Dekker: New York, 1976; Vol. 9.

(22) R. S. Sirohi and M. A. Gensham, J. Electrochem. Soc. 1969, 116, 910.
Table 1. Electrochemical and SR-XRF Dataa

$\begin{array}{lccccc}\begin{array}{c}\text { modification } \\ \text { techniques }\end{array} & \begin{array}{c}\text { charge } \\ (\mu \mathrm{C})\end{array} & \begin{array}{c}\text { surface } \\ \text { concn } \\ \left(\mu \mathrm{g} \mathrm{cm}^{-2}\right)\end{array} & \begin{array}{c}\text { mean } \alpha \\ \mathrm{Co} \alpha\end{array} & \begin{array}{c}\sigma_{\mathrm{Co}} / I_{\mathrm{C}} \\ \times 100(\%)\end{array} & \begin{array}{c}\text { total surface } \\ \text { derived by }\left(\mu \mathrm{g} \mathrm{cm}^{-2}\right)\end{array} \\ \text { immersion } & 2.4 \pm 0.2 & 0.074 & 135.4 & 28.4 & 0.08 \pm 0.02 \\ \text { drop drying } & 3.2 \pm 0.2 & 0.098 & 192.7 & 37.4 & 0.14 \pm 0.05 \\ \text { potential cycling } & 3.6 \pm 0.2 & 0.110 & 2036.7 & 31.6 & 3.3 \pm 1.0\end{array}$

${ }^{a}$ The first three columns show the electrochemical data concerning the three modification techniques: the charge due to the $\mathrm{Co}$ (III)/Co(II) reduction peak and the calculated cobalt surface concentration. The last three columns show the SR-XRF data, respectively, the mean Co $\mathrm{K} \alpha$ intensity, its relative standard deviation, and the total cobalt surface concentration.

$0.3 \mu \mathrm{C}$, which corresponds to an adsorbed Co surface density of $\sim 0.074 \mu \mathrm{C} \mathrm{cm}^{-2}$ (Table 1).

A similar electrochemical behavior is shown when a modified electrode is created by the drop drying method. Figure $1 \mathrm{~b}$ represents the evolution of the $\mathrm{Co}_{0}$ (III)/Co(II)-related adsorption peaks during the first 10 scans when the modified CoTSPc gold electrode is scanned in a blank buffer solution after the drop drying process. The charge under the $\mathrm{Co}$ (III) $/ \mathrm{Co}$ (II) adsorption peak, again based on scan 20 , is equal to $3.2 \pm 0.2 \mu \mathrm{C}$, which 

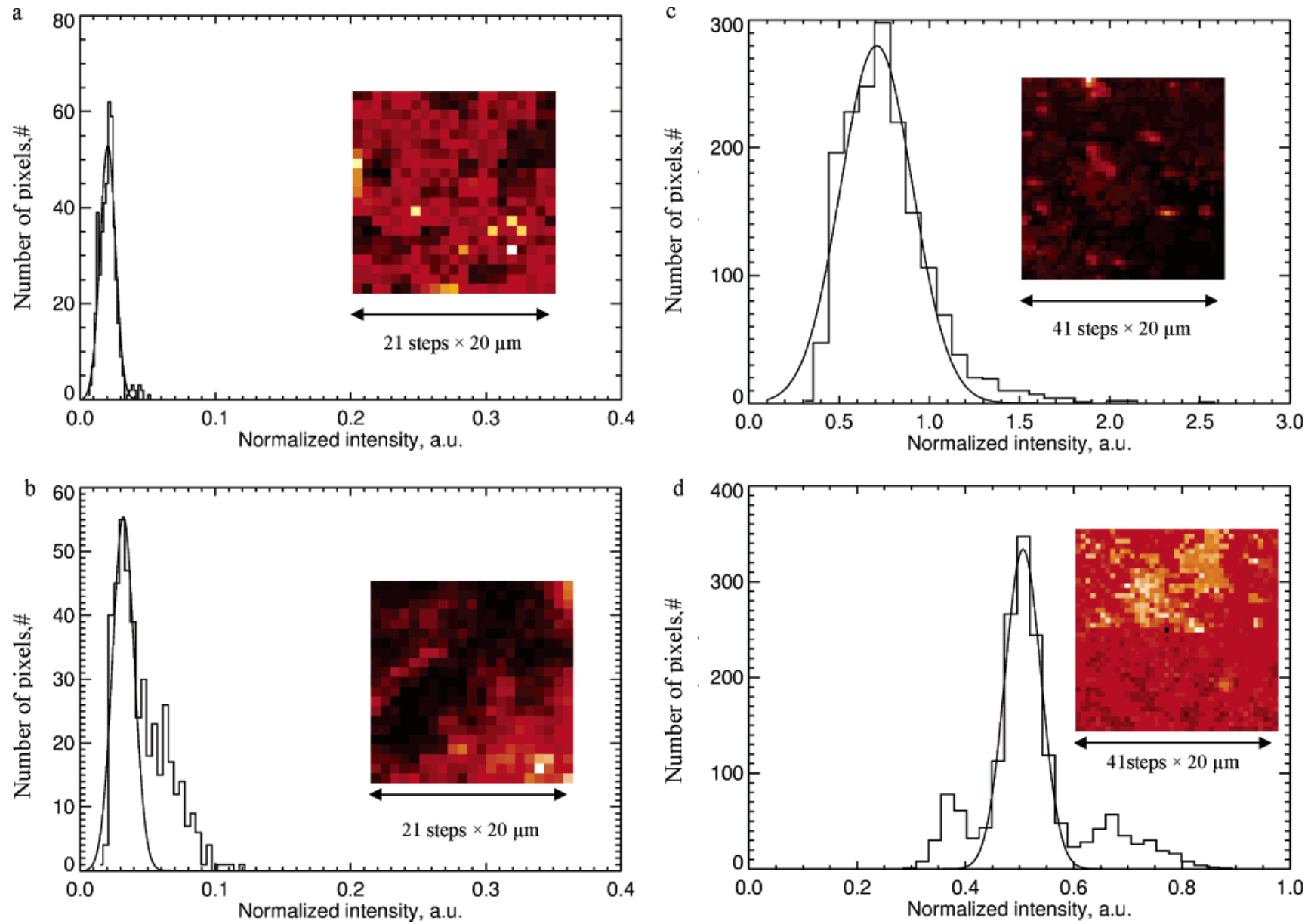

Figure 3. Elemental map and intensity distribution of cobalt on a gold electrode modified by three different modification techniques: electrode immersion (a), drop drying (b), and potential cycling (c and d). The XRF maps consists of a $21 \times 21$ or $41 \times 41$ raster using $20-\mu \mathrm{m}$ step size in both $X$ and $Y$ directions.

corresponds to a Co surface density of $0.098 \mu \mathrm{C} \mathrm{cm}{ }^{-2}$ (Table 1 ). Comparison of the two above-mentioned modification techiques leads to the conclusion that more CoTSPc is adsorbed onto the electrode surface when modifying by drop drying. This is due to the intensive contact between the droplet and the electrode and the intensive drying process.

The recorded cyclic voltammogram corresponding to the potential cycling method is shown in Figure 1c. The first scan already shows that the charges of the adsorption processes reach their maximum value. This means that there is no change in the surface condition, as will be explained below when dealing with the spectroscopic analysis of the modified electrodes. The charge under the $\mathrm{Co}$ (III) $/ \mathrm{Co}$ (II) adsorption reduction peak is equal to $3.6 \pm 0.2 \mu \mathrm{C}$, which corresponds to an adsorbed Co surface concentration value of $0.110 \mu \mathrm{C} \mathrm{cm}^{-2}$ (Table 1). Curves 2-7 show that a new oxidation peak shifts from 0.3 to $0.45 \mathrm{~V}$ versus SCE during the first 20 scans. This oxidation peak has previously been explained in the literature ${ }^{14}$ and can be attributed to the ring oxidation of CoTSPc present in solution on a Co(III)TSPc-modified gold electrode. The shift in peak potential is probably due to the difference in solution, a CoTSPc pH 12 buffer solution versus a blank $\mathrm{pH} 12$ buffer solution.

Micro SR-XRF measurements were performed to provide further experimental evidence of the formation of an adsorbed CoTSPc layer at the gold electrodes and to study the uniformity of the formed thin film quantitatively. Figure 3 shows the measured elemental maps and the corresponding intensity distribution of the detected $\mathrm{Co}_{\mathrm{K}} \mathrm{K}$ (Figure 3a, immersion; b, drop drying; $c$ and d, potential cycling) obtained by SR-XRF measurements after normalization with the gold $\mathrm{M}$ line. These figures not only provide evidence of the occurrence of adsorbed CoTSPc but also give an estimate of the amount of adsorbed CoTSPc. The discrepancy between the values for the surface concentration for both methods can be explained by the fact that only CoTSPc monomer species and not dimers can be electrochemically detected..$^{23}$ It is expected that CoTSPc dimers surround the modified electrode in any circumstance, but these compounds are not electrochemically active. In contrast, the XRF measurements are able to detect all kind of cobalt species, monomers as well as dimers. Therefore, the XRF signal includes both the monomer and the dimer signal leading to significant higher amounts of cobalt compared to the electrochemically detectable amount (only monomers).

On the basis of Figure 3, it can be concluded that when cycling the gold electrode in a $\mathrm{Co}$ (II)TSPc $\mathrm{pH} 12$ buffer solution during 100 scans in a potential window from -1.2 to $0.6 \mathrm{~V}$ versus SCE (Figure 3c), the amount of adsorbed CoTSPc is much larger than is the case for the two other techniques (Figure $3 \mathrm{a}$ and $\mathrm{b}$ ). The

(23) Peeters, K.; De Wael, K.; Vincze, L.; Falkenberg, G.; Adriaens, A. Article in preparation. 
normalized intensity is much higher (more than a 10-fold) in the case of potential cycling. Relative standard deviation and calculated Co surface concentration values are presented in Table 1.

A similar experiment was performed to show the influence of cycling in a blank buffer solution during 20 scans (after the modification) on the homogeneity of the adsorbed CoTSPc layer. A gold electrode modified by recording 100 voltammetric scans in a pH 12 buffer solution containing $6 \times 10^{-3} \mathrm{~mol} \mathrm{~L}^{-1} \mathrm{Co}$ (II)TSPc was rinsed with buffer solution and directly analyzed by SRXRF. The scanning in a $\mathrm{pH} 12$ buffer solution after the modification was now skipped in the procedure. The elemental map and the matching frequency distribution of $\mathrm{Co} \mathrm{K} \alpha$, obtained by SR-XRF measurements after normalization with the gold $\mathrm{M}$ line, are presented in Figure 3d. The histogram shows a more heterogeneous distribution of Co. This means that the scanning step in the buffer solution (after modification) is needed to obtain a homogeneous adsorbed CoTSPc layer. This hypothesis can also be used to explain the increase of the peak current with growing scan number for the adsorption processes in Figure 1a and b. The increase reflects the transformation of a heterogeneous to a more homogeneous surface condition. The enhancement of homogeneity can be attributed to the fact that by cycling the potential the deposited layer goes through different orientation stages until a thicker and more stable layer is formed. In the latter situation, the flipping process from one orientation into another is no longer possible.

On the basis of the above-mentioned arguments, it can be concluded that a more homogeneous CoTSPc-modified gold electrode is created when cycling in a Co(II)TSPc pH 12 buffer solution during 100 scans. The homogeneity is clearly enhanced by the subsequent scanning in the buffer solution after the modification. As there is more CoTSPc adsorbed onto the surface, the deposition is more efficient. More information on this modification technique, also called electrodeposition, and on its use to create modified electrodes with important electrocatalytic properties can be found in the literature..$^{12}$

Dimerization of Co(II)TSPc. The authors also observed that the efficiency of electrodeposition highly depends on the degree of dimerization of $\mathrm{Co}$ (II)TSPc. Prior to explaining this influence, the monomer-dimer equilibrium was studied. UV-visible absorption spectra of tetrasulfonated phthalocyanines have been described in terms of the monomer-dimer equilibrium. ${ }^{9-11}$ The absorption spectrum is dominated by the Q-band arising from $\pi-\pi^{*}$ transitions. Within the Q-band, several peaks can be assigned to either free molecules or aggregates. In general, the aggregate peak is blue-shifted with respect to the monomer peak. Aggregation can be influenced by the $\mathrm{pH}$, ionic strength, solvent, etc. Figure 4 shows the UV-visible spectra obtained from the $\mathrm{Co}$ (II)TSPc samples, obtained from three different manufacturers, in a $\mathrm{pH} 12$ buffer solution in a concentration range from $2.5 \times$ $10^{-6}$ to $2 \times 10^{-5} \mathrm{~mol} \mathrm{~L}^{-1}$. In this concentration range, a linear relationship between absorbance and concentration is visible. As the Lambert-Beer equation is no longer valid at a value of 3 for the absorbance, only a hypothesis can be formulated for the concentrations that give rise to such values for the absorbance. The UV-visible measurements of the three CoTSPc samples were performed in order to compare the monomer-dimer equilibrium.
The monomer peak for the Co(II)TSPc species in a pH 12 buffer solution is located at $661 \mathrm{~nm}$, while the dimer peak is observed at $634 \mathrm{~nm} \cdot{ }^{10-11}$ Figure $4 \mathrm{a}$ shows that the $\mathrm{pH} 12$ buffer solution containing CoTSPc powder purchased from Rhodes University of Grahamstown consists of monomer species as well as dimers in a concentration range from $2.5 \times 10^{-6}$ to $2 \times 10^{-5}$ mol L ${ }^{-1}$. Because of the higher absorbance for the monomer peak in the complete concentration range, it can be stated that more monomers are present even at higher concentrations (e.g., $6 \times$ $10^{-3} \mathrm{mmol} \mathrm{L}^{-1}$ for the modification technique). As can be seen in Figure $4 \mathrm{~b}$, the sample purchased from Midcentury consists of only a small percentage of dimers. The Co(II)TSPc species can be found in principle in their monomer form. Therefore, it is evident that, for higher concentrations, Co(II)TSPc will be present mainly in its monomer form. In contrast, the UV-visible spectrum of Co(II)TSPc purchased from Porphyrin Systems (Figure 4c) reveals that for the highest concentrations of CoTSPc more dimers than monomers are present in the $\mathrm{pH} 12$ buffer solution. On the basis of this observation, it can be concluded that, at high concentrations, such as $6 \times 10^{-3} \mathrm{mmol} \mathrm{L}^{-1}$, aggregation will be completed and dimers are the dominant species.

When comparing the UV-visible spectra of all Co(II)TSPc samples, it is clear that the monomer-dimer equilibrium is different. Based on Figure 4, it is assumed that at high $\mathrm{Co}$ (II)TSPc concentrations (e.g., $6 \times 10^{-3} \mathrm{mmol} \mathrm{L}^{-1}$ for the modification technique) more dimers than monomers are formed in the case of Porphyrin Systems. For the CoTSPc sample, purchased from Midcentury and Rhodes University of Grahamstown, the figures show that even at high CoTSPc concentrations the monomers are in majority. In the next section, the influence of the monomerdimer equilibrium on the electrochemical behavior at gold electrodes will be discussed.

Influence of the Dimerization of CoTSPc on the Electrodeposition at Gold Surfaces. As described in the first section, the most efficient modification technique that leads to a homogeneous CoTSPc layer onto the gold surface is achieved by recording successive cyclic voltammetric scans (100 scans) in a potential window from -1.2 to $0.6 \mathrm{~V}$ versus SCE in a $\mathrm{pH} 12$ buffer solution containing $6 \times 10^{-3} \mathrm{~mol} \mathrm{~L}^{-1} \mathrm{Co}$ (II)TSPc. The modification was now done for all CoTSPc samples.

The modification of gold electrodes with CoTSPc (Midcentury) has previously been described in detail in the literature, ${ }^{14}$ including the explanation of all peaks recorded in the corresponding CoTSPc $\mathrm{pH} 12$ buffer solution. It was observed ${ }^{13}$ that the mechanism of the deposition can be considered as a multistep process. The initial deposition, from scan 1 to the breaking point (point of maximum adsorption), is controlled by kinetics resulting in a chaotic multilayer. After the breaking point, a slow reorganization of the chaotic multilayer occurs. The scan related to the breaking point is shown in Figure 5. It could be observed that the charge under the peaks associated with $\mathrm{CoTSPc}_{\mathrm{ads}}$ (taken at the breaking point, when deposition is virtually complete) is equal to $4.1 \pm 0.2 \mu \mathrm{C}$. As already suggested by the differences in the measured UVvisible spectra corresponding to $\mathrm{Co}$ (II)TSPc from Porphyrin Systems and Rhodes University of Grahamstown (Figure 4), a different electrochemical behavior is expected when using $\mathrm{Co}_{-}$ (II) TSPc from these suppliers. 

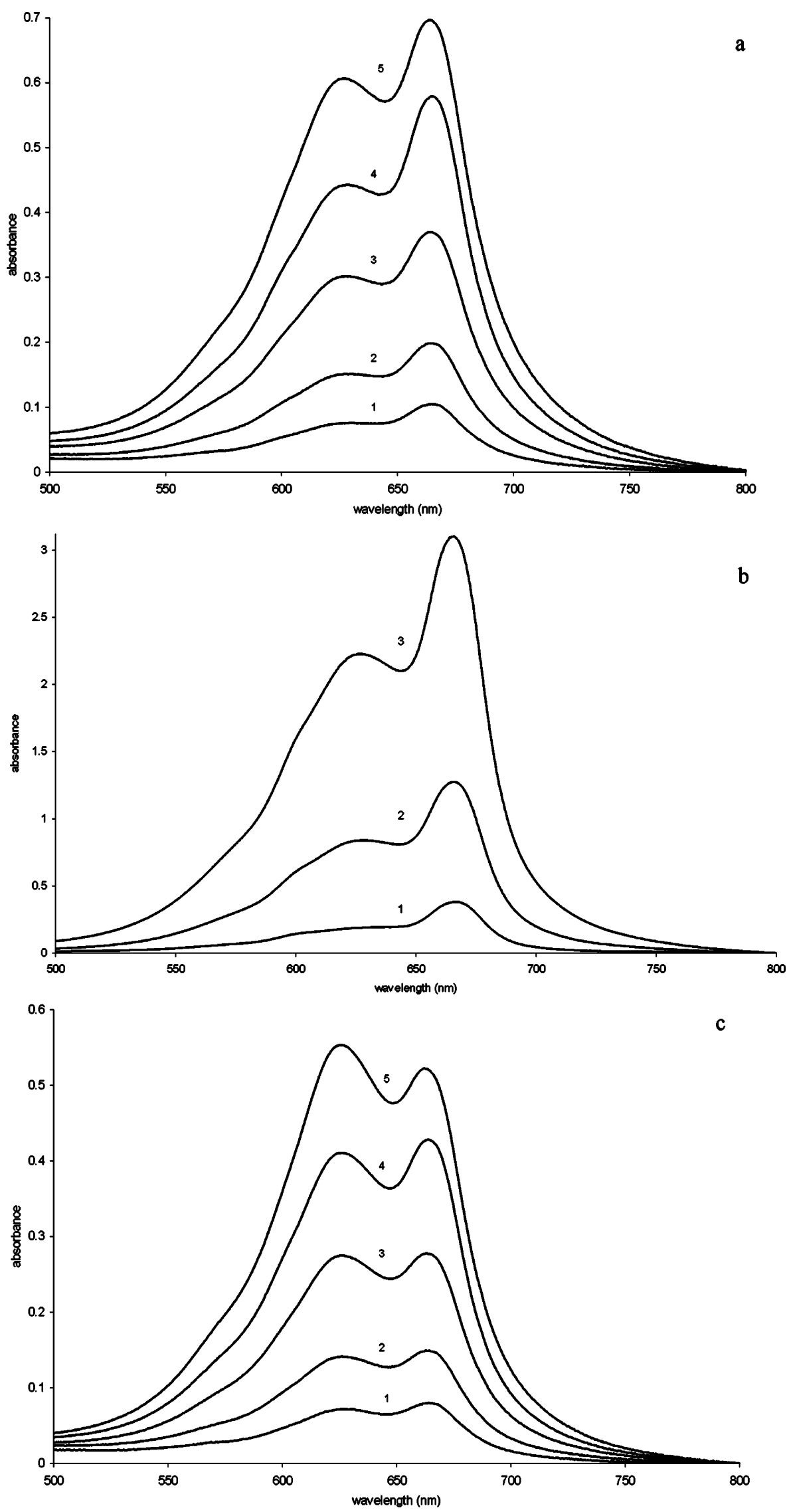

Figure 4. Absorbance spectra of a pH 12 buffer solution containing different cobalt(II) tetrasulfonated phthalocyanine concentrations purchased from the following. (a) Rhodes University: (1) $2.5 \times 10^{-6}$, (2) $5.0 \times 10^{-6}$, (3) $1.0 \times 10^{-5}$, (4) $1.5 \times 10^{-5}$, and (5) $2.0 \times 10^{-5} \mathrm{~mol} \mathrm{\textrm {L } ^ { - 1 }}$. (b) Midcentury: (1) $4.4 \times 10^{-6}$, (2) $1.9 \times 10^{-5}$, and (3) $4.8 \times 10^{-5} \mathrm{~mol} \mathrm{~L}^{-1}$. (c) Porphyrin Systems: (1) $2.5 \times 10^{-6}$, (2) $5.0 \times 10^{-6}$, (3) $1.0 \times 10^{-5}$, (4) $1.5 \times 10^{-5}$, and (5) $2.0 \times 10^{-5} \mathrm{~mol} \mathrm{~L}^{-1}$. 


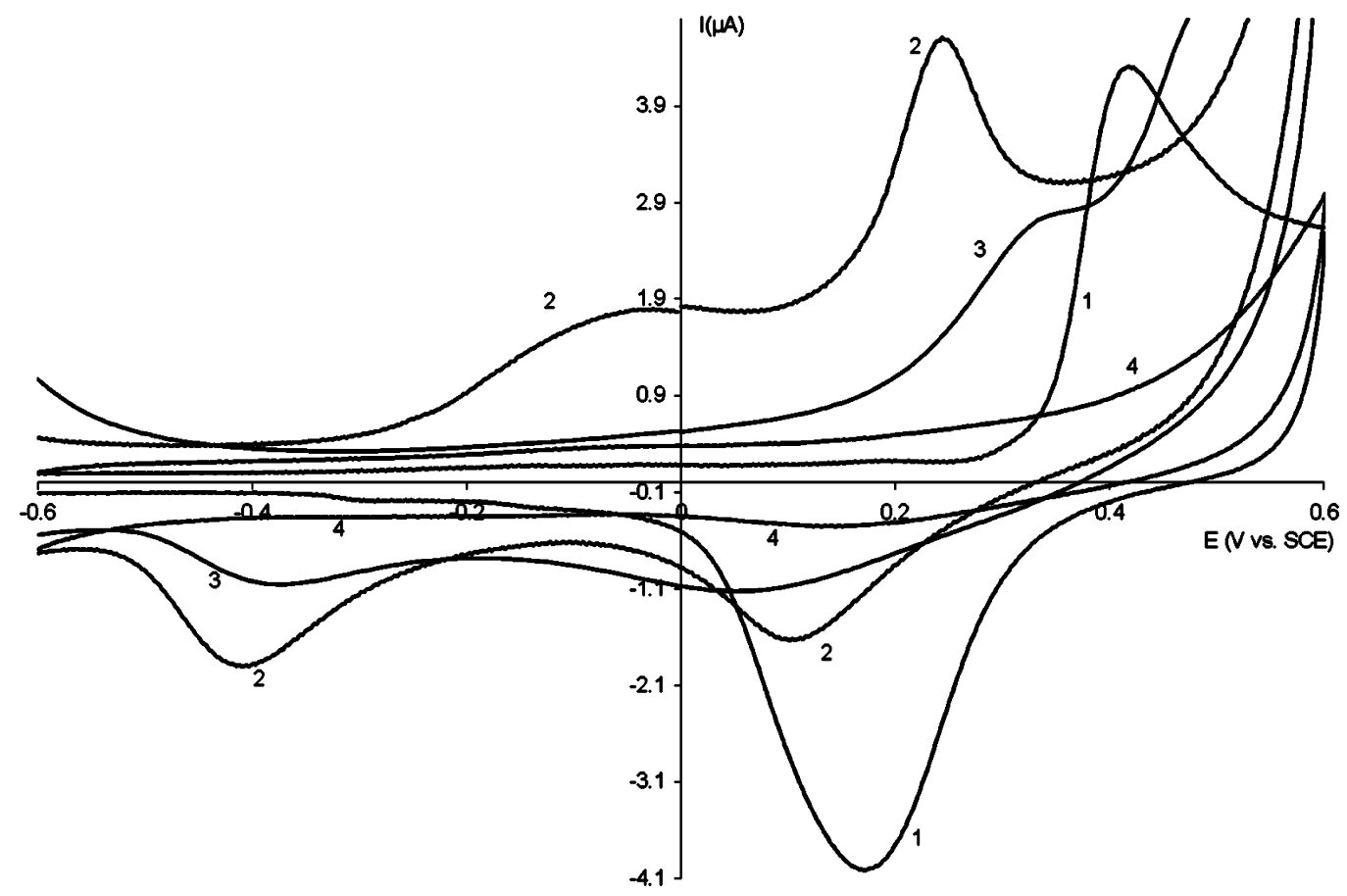

Figure 5. Current-potential curves recorded at a gold electrode in a $\mathrm{pH} 12$ buffer solution (1) and the voltammetric scan measured at the breaking point for the three different companies: Midcentury (2), Rhodes University (3), and Porphyrin Systems (4).

Curve 2 in Figure 5 represents the voltammetric scan related to the breaking point recorded in a $\mathrm{pH} 12$ buffer solution containing $6 \times 10^{-3} \mathrm{~mol} \mathrm{~L}^{-1} \mathrm{Co}$ (II)TSPc, purchased from Rhodes University of Grahamstown. The charge under the peak associated with $\mathrm{CoTSP}_{\mathrm{ads}}$ reduction is equal to $3.7 \pm 0.2 \mu \mathrm{C}$. This value for the charge, corresponding to the amount of adsorbed CoTSPc $\left(1.96 \mu \mathrm{g} \mathrm{cm}^{-2}\right)$, is somewhat lower than the value obtained from the Midcentury sample. This discrepancy can be explained by the difference in monomer-dimer equilibrium. Compared to CoTSPc from Rhodes University of Grahamstown, the monomerdimer ratio is much higher in the case of Midcentury. As the amount of adsorbed CoTSPc is higher in the case of Midcentury, it can be concluded that only the monomer species of CoTSPc can be adsorbed onto the gold surface.

Curve 3 in Figure 5 corresponds to scan 20 recorded in a $6 \times$ $10^{-3} \mathrm{~mol} \mathrm{~L}^{-1} \mathrm{Co}$ (II)TSPc buffer solution purchased from Porphyrin Systems. As shown in Figure 4c, the UV-visible spectrum indicates that principally dimers are present in the solution. Because the adsorption onto electrodes only takes place through the monomer species, no electrochemical adsorption reactions can be obtained. The electrode is surrounded by dimers; however, there is no specific bonding between the two resulting in an electrochemical signal.

\section{CONCLUSIONS}

A comparison of different surface modification techniques for electrodes is described in this article. Performing successive cyclic voltammetric scans leads to a more efficient deposition, which results in a uniformly adsorbed CoTSPc layer. A study of the deposited CoTSPc layer by scanning micro SR-XRF allowed us to derive the adsorbed Co surface concentration values and their relative standard deviations for the various surface modification techniques, which could be used to establish the optimum electrochemical deposition conditions for CoTSPc.

It was also observed that, depending on the monomer-dimer equilibrium of the $\mathrm{Co}$ (II)TSPc solution, the electrochemical deposition can be influenced. This article shows that only the monomer species of CoTSPc can be adsorbed electrochemically onto a gold surface.

\section{ACKNOWLEDGMENT}

This work was supported by the European CommunityResearch Infrastructure Action under the FP6 "Structuring the European Research Area" Programme (through the Integrated Infrastructure Initiative "Integrating Activity on Synchrotron and Free Electron Laser Science"). The authors gratefully acknowledge A. Madder and A. Gea (UGent, Belgium) for giving us the opportunity to perform the UV-visible measurements.

Received for review May 12, 2005. Accepted June 17, 2005.

AC0508245 